\title{
Calculations of Dark Current in Interband Cascade Type-II Infrared InAs/GaSb Superlattice Detector
}

\author{
K. Hackiewicz*, P. Martyniuk, J. Rutkowski and A. Kowalewski \\ Institute of Applied Physics, Military University of Technology, S. Kaliskiego 2, 00-908 Warsaw, Poland \\ (Received May 4, 2017; in final form August 29, 2017)

\begin{abstract}
In this paper we investigate interband cascade type-II mid-wavelength infrared InAs/GaSb superlattice detector in temperature range from $200 \mathrm{~K}$ to $300 \mathrm{~K}$. The paper is based on the theoretical calculation of dark current treated as a sum of two components: average bulk current and average leakage current, flowing through the device. The average leakage current results from a comparison of theoretically calculated bulk current and measured one. We show that it is possible to fit theoretical model to experimental data, assuming that transport in absorber is determined by the dynamics of the intrinsic carriers. Based on the fit we estimated carrier lifetime greater than $100 \mathrm{~ns}$ in temperature range $200-300 \mathrm{~K}$.
\end{abstract}

DOI: 10.12693/APhysPolA.132.1415

PACS/topics: 73.40.Mn, 73.61.Ey, 78.30.Fs, 85.60.Bt, 85.60.Gz

\section{Introduction}

In recent years, there have been strong efforts in the development of photodetectors sensitive to infrared radiation. One of the most important steps in the fabrication process of such photodetector is the proper design of the structure, so that the detectors are able to correctly operate at room temperatures or temperatures reached by thermoelectric coolers. Such devices have a magnitude of applications. The current development of infrared detection generally allows to choose between the two groups of materials for infrared detectors: detectors based on IIVI materials (mercury cadmium telluride) [1] and III-V materials $[2,3]$. In the second group of materials intersubband quantum well infrared photodetectors (QWIPs) and materials such as InAs, GaSb, AlSb and their related alloys can be distinguished. InAs/GaSb superlattice is now considered as a new material system for the fabrication of infrared photodetectors, which may be able to achieve better performance than mercury cadmium telluride. Moreover, the cascade configuration allows a more efficient use of the incident photons because the absorbers can be kept shorter than the carrier diffusion length and in this type of photodetectors the absorption efficiency is effectively increased. In interband cascade photodetectors (ICPs) each stage is divided into three regions according to the processes as follows: (1) absorption, (2) carrier relaxation and (3) tunneling transport, as shown in Fig. 1. In ICPs carriers flow in one direction depending on its type. This is achieved with the use of unipolar barriers. The individual absorber is sandwiched between wider bandgap electron and hole barriers. The relaxation region consists of multiple QWs with energy levels, which form an energy ladder for directing and facilitating elec-

\footnotetext{
* corresponding author; e-mail: klaudia.hackiewicz@wat.edu.pl
}

tron transport to the right. The conduction bands and the valence bands of adjacent absorbers are connected in series using an interband tunneling heterostructure [4].

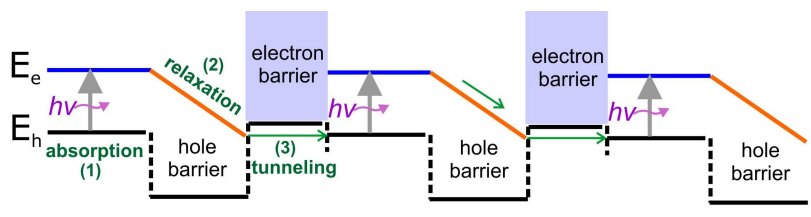

Fig. 1. General schematic for interband cascade detectors.

In this paper, we report on interband cascade midwavelength infrared (MWIR) detector based on type-II InAs/GaSb superlattice (T2SL). We present performance, based on current-voltage $(I-U)$ characteristics and spectral characteristics obtained for such cascade structures. Moreover, theoretical calculations of dark current in multi-stage interband detector are presented. Dark current in this paper is treated as a sum of two components: average bulk current and average leakage current. We compare this theoretical data to experimental results and discuss about the potential performance of such detectors.

\section{Device design and fabrication}

Examined structure was fabricated in Center for High Technology Materials, University of New Mexico, Albuquerque, New Mexico. The device was grown on Tedoped (100) GaSb substrate using molecular beam epitaxy. The detailed growth procedure is described by Gautam et al. [4]. The schematics of the measured and described detector is illustrated in Fig. 2.

The device consists of 120 periods of 12.5 monolayers (12.5 MLs) of InAs/5 ML AlSb as the bottom contact layer. This layer is followed by relaxation region. The total thickness of each transport region is $75 \mathrm{~nm}$. All the layers in the barriers and absorbers are nonintentionally doped regions. In the analyzed tempera- 
ture range from $200 \mathrm{~K}$ to $300 \mathrm{~K}$, the transport in absorber is determined by the dynamics of intrinsic carriers, e.g. $n_{i}(300 \mathrm{~K})=8.87 \times 10^{21} \mathrm{~m}^{-3}[6]$. Each absorber is composed of a 28 periods InAs/GaSb (nine/nine monolayers) T2SL. The total thickness of each absorber $d$ is about $153 \mathrm{~nm}$. Diffusion length is expected to be longer than the absorber thickness. The absorbers for each stage are made identical in order to keep the material growth simple. Relaxation region, barrier region and absorber form a single-stage interband cascade region. Investigated structure consists of seven such areas. The structure is terminated by GaSb:Be which formed a $p$-type contact layer.

The measured detectors were the same size, the electrical detector area is $1.60 \times 10^{-7} \mathrm{~m}^{2}$ while the optical area is equal to about $3.14 \times 10^{-8} \mathrm{~m}^{2}$.

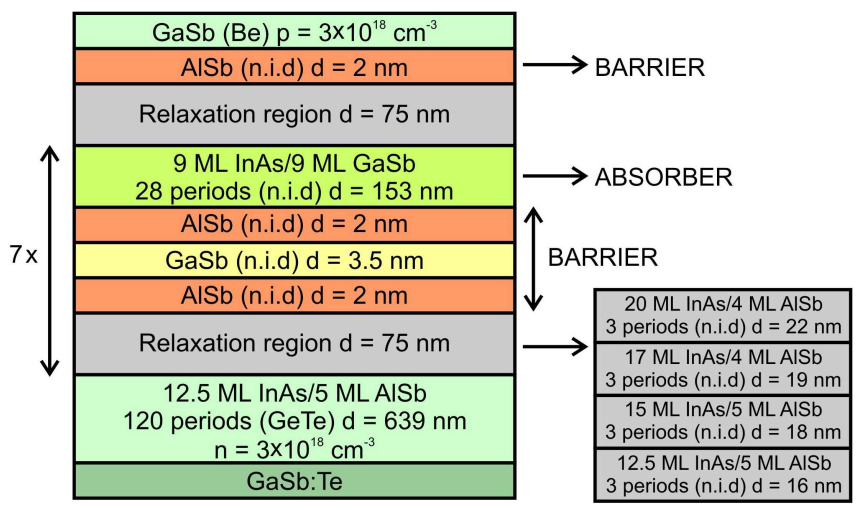

Fig. 2. Structural schematic of the MWIR T2SL cascade detector.

\section{Experimental results}

\subsection{Spectral characteristics}

The spectral responsivity of investigated detector has been observed in temperature range of $T=200-300 \mathrm{~K}$. These characteristics are shown in Fig. 3. The Fourier transform infrared (FTIR) spectrometer was used to perform the spectral measurements. The peak responsivity for $T=200 \mathrm{~K}$ and $T=300 \mathrm{~K}$ reaches about $0.25 \mathrm{~A} / \mathrm{W}$ and $0.2 \mathrm{~A} / \mathrm{W}$, respectively. Based on the graph below it can be seen that tested detector has an extremely low sensitivity compared to the values published in the literature [7]. Furthermore, the responsivity of the detector decreases slightly with increasing temperature, which is related to the change in the diffusion length.

The cut-off wavelength was not found to be changing significantly for unbiased and biased conditions. This parameter is decreasing with the temperature assuming $\lambda_{c}=5.24 \rightarrow 4.81 \mu \mathrm{m}$ at $300 \mathrm{~K}$ and $200 \mathrm{~K}$, respectively. We use these values for the bandgap calculations. The variation of the bandgap of T2SL InAs/GaSb with temperature is described by linear-quadratic relation proposed by Varshni [8]:

$$
E_{g}(T)=E_{g 0}-\frac{\alpha T^{2}}{T+T_{0}},
$$

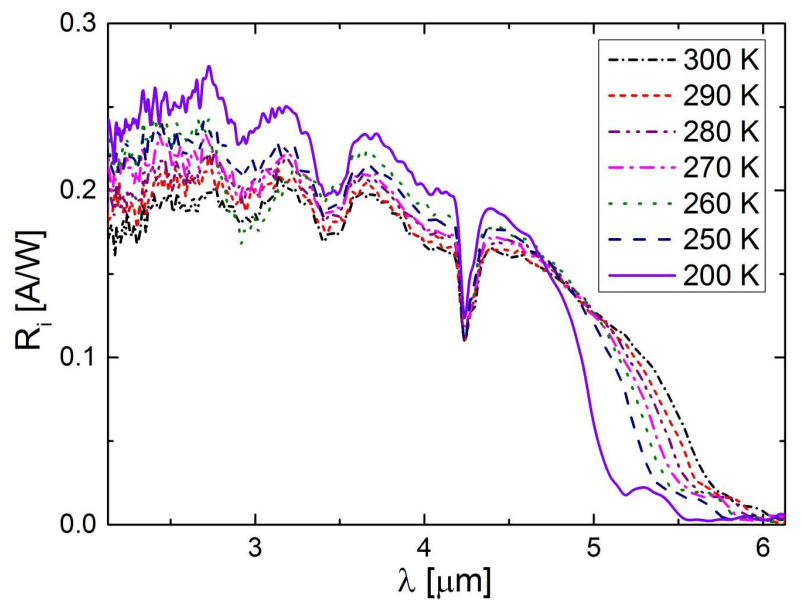

Fig. 3. Current responsivity of T2SL InAs/GaSb MWIR cascade detector at zero bias voltage.

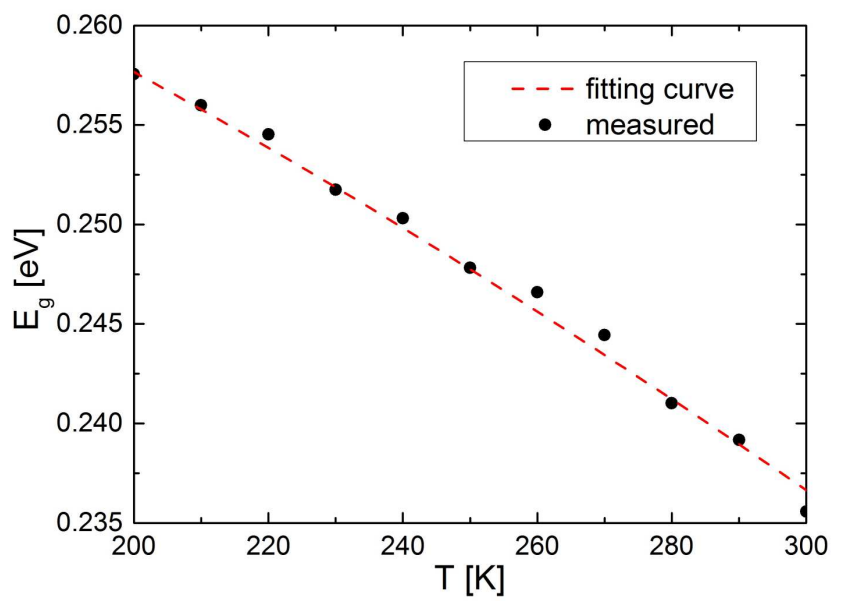

Fig. 4. Fitting curve using the Varshni equation: bandgap versus temperature for T2SL InAs/GaSb cascade detector.

where $T_{0}$ is a constant $[\mathrm{K}], E_{g 0}$ is the width of the semiconductor bandgap at $0 \mathrm{~K}[\mathrm{eV}], \alpha$ is a fitting parameter $[\mathrm{eV} / \mathrm{K}]$ and $T$ is the temperature $[\mathrm{K}]$. The fitting parameters in the Varshni formula are assumed to be equal to $E_{g 0}=0.28 \mathrm{eV}, \alpha=4 \times 10^{-4} \mathrm{eV} / \mathrm{K}$ and $T_{0}=550 \mathrm{~K}$. Figure 4 shows the dependence of energy bandgap on temperature. Solid lines are plotted using the Varshni formula.

\subsection{Electrical characteristics}

The dark current characteristics as a function of bias voltage for selected temperatures in the range from $200 \mathrm{~K}$ to $300 \mathrm{~K}$ are shown in Fig. 5 . For the bias voltage value of $-0.01 \mathrm{~V}$, at temperature $300 \mathrm{~K}$, the dark current is equal to $3.19 \times 10^{-5} \mathrm{~A}$, while at temperature $200 \mathrm{~K}$, the dark current reaches $2.46 \times 10^{-6} \mathrm{~A}$. A dark current is sensitive to a bias voltage across entire range of applied reverse bias and does not saturate for all investigated temperatures. We think that high value of dark current is the result of defects-assisted tunneling and large surface currents (leakage currents). 


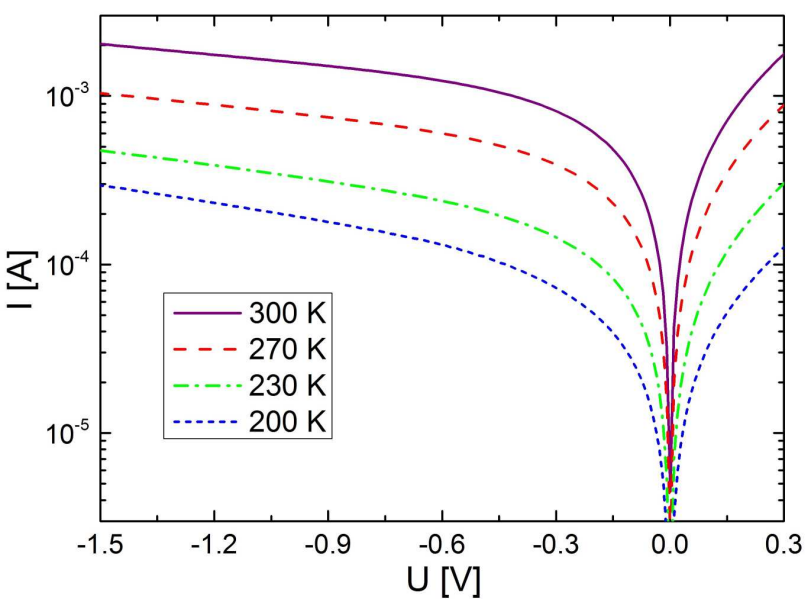

Fig. 5. $I-U$ characteristics of T2SL InAs/GaSb MWIR cascade detector.

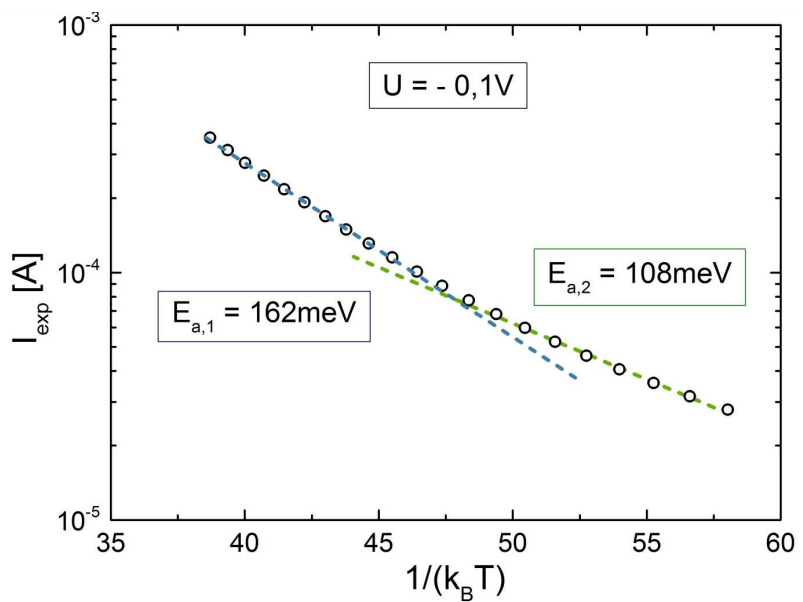

Fig. 6. Characteristic of dark current versus temperature at $-0.1 \mathrm{~V}$ of applied bias.

Figure 6 shows the Arrhenius plot of dark current versus temperature at $-0.1 \mathrm{~V}$ of applied bias. It is shown that at high temperature range, between $250 \mathrm{~K}$ and $300 \mathrm{~K}$, an activation energy is higher than at low temperature range, between $245 \mathrm{~K}$ and $200 \mathrm{~K}$. The activation energy of $162 \mathrm{meV}\left(\approx E_{g} / 2\right)$ indicates that in this temperature range $(250-300 \mathrm{~K})$ the dominant dark current mechanism is a Shockley-Read-Hall (SRH) recombination. At low temperature range (200-245 K) an activation energy of $108 \mathrm{meV}$ is lower than $E_{g} / 2$ and it typically indicates that current flows through leakage channels. Activation energies are estimated for different values of applied bias and for all of those voltages are almost the same.

\section{Modeling of current-voltage characteristics}

We make an effort to model and thus clarify the $I-U$ characteristics simultaneously comparing it with the experimentally measured. In order to explain $I-U$ characteristics of ICPs the theoretical dark current model proposed by Hinkey and Yang is used [9]. The transport region plays a role similar to the depletion region in a conventional $p-n$ junction to direct current in one direction. Therefore, the average bulk dark electrical current that flows through the device is given by Eq. (2). Because the investigated structure has seven identical stages $\left(N_{s}=7\right)$, it should be noted that the current flowing through each of them will be equal.

$$
\begin{aligned}
& I_{b}(U)=2 e n_{i} L \tau^{-1} \sum_{i=1}^{N_{s}} \frac{1}{N_{s}} \frac{\sinh (d / L)}{\cosh (d / L)} \\
& \quad \times\left(\exp \left(\frac{e U}{\beta N_{s} k_{\mathrm{B}} T}\right)-1\right),
\end{aligned}
$$

where $e, L, \tau, k_{\mathrm{B}}$ and $\beta$ are elementary electric charge, ambipolar diffusion length, carrier lifetime, the Boltzmann constant and diode ideality factor, respectively. Diffusion length and diffusion coefficients are connected with carrier lifetime $\tau$ by the Einstein relation. The ambipolar diffusion length is given by

$$
L=L_{e}=L_{h}=\sqrt{D \tau} .
$$

The ambipolar diffusion coefficient $D$ is defined as:

$$
D=\frac{2 D_{e} D_{h}}{D_{e}+D_{h}}
$$

where $D_{e}$ and $D_{h}$ are electron and hole diffusion coefficients, respectively. Both diffusion coefficients can be expressed by

$$
D_{e, h}=\frac{\mu_{e, h} k_{\mathrm{B}} T}{e},
$$

where $\mu_{e}$ and $\mu_{h}$ are electron and hole mobility, respectively. Carrier mobilities of InAs/GaSb T2SL were measured by several authors and these values depend on the accuracy of the measurement method [6, 10-12]. We assume $\mu_{e}=0.1 \mathrm{~m}^{2} /(\mathrm{V} \mathrm{s})$ and $\mu_{h}=0.01 \mathrm{~m}^{2} /(\mathrm{V} \mathrm{s})$. Parameter $\beta$ is estimated at 1.22 , because at this value we obtain the best fitting level in forward bias direction.

Based on the estimated values of the energy gap from the Varshni formula (see Eq. (1)) we calculate theoretical intrinsic carrier concentration from the following relation:

$$
n_{i}=2\left(\frac{2 \pi k_{\mathrm{B}}}{h^{2}}\right)^{1.5}\left(m_{e}^{*} m_{h}^{*}\right)^{0.75} T^{1.5} \exp \left(\frac{E_{g}}{2 k_{\mathrm{B}} T}\right) .
$$

Effective electron $m_{e}^{*}$ and hole $m_{h}^{*}$ masses are calculated according to

$$
m_{e, h}^{*}=\sqrt[3]{m_{e, h \|}^{*} m_{e, h \perp}^{*}}
$$

where in-plane $m_{e, h \|}^{*}$ and growth $m_{e, h \perp}^{*}$ direction effective masses for this structure are equal to: $m_{e \|}^{*}=$ $0.0235 m_{0}, m_{e \perp}^{*}=0.0275 m_{0}, m_{h \|}^{*}=0.0342 m_{0}$ and $m_{h \perp}^{*}=79.8 m_{0}[13]$.

Because the tunneling effects are not included in our theoretical calculation of bulk current $I_{b}$ we compare those results with experimentally measured current and value of average leakage current $I_{s}$. The current that flows through the structure is given by

$$
I=I_{b}+I_{s} .
$$

As can be seen from the above formulae, the correct fit of the theoretical $I-U$ characteristics to the experimentally measured requires knowledge of the carrier lifetime. The 
correct estimation of carrier lifetime is very difficult. For this purpose, simultaneously with the change of carrier lifetime the dependence of leakage current $I_{s}$ on bias voltage $U$ was investigated. The existence of leakage currents leads to the linear dependence on bias voltage. Desired character of changes is obtained only for a narrow range of $\tau$ values. Figure 7 shows the change in the leakage current as a function of applied voltage for different carrier lifetimes at $300 \mathrm{~K}$. The best value of carrier lifetime at $300 \mathrm{~K}$ is equal to $108 \mathrm{~ns}$. The present character of changes is examined for temperature range from $200 \mathrm{~K}$ to $300 \mathrm{~K}$.

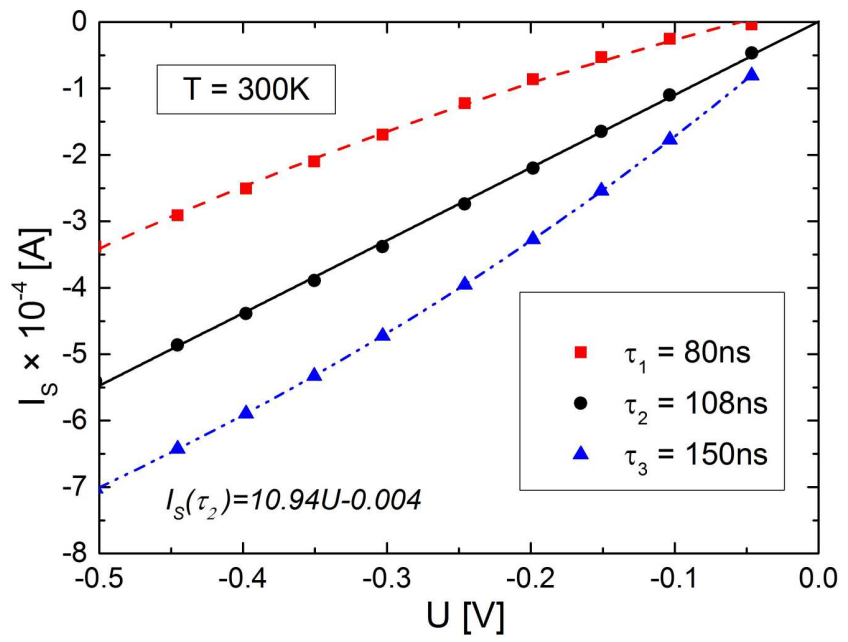

Fig. 7. Leakage current as a function of applied voltage for three different carrier lifetimes at $300 \mathrm{~K}$ with trend line equation for $\tau_{2}=108 \mathrm{~ns}$.

\section{Results}

Figure 8 shows the magnitude of the bulk current and leakage current, which affects the shape of the total dark current flowing through the device at $200 \mathrm{~K}$ and $300 \mathrm{~K}$. The dots in Fig. 8 show experimentally measured points which correspond to Fig. 5. As can be seen, in the voltage range from $-0.5 \mathrm{~V}$ to $0.1 \mathrm{~V}$ experimental results do not vary significantly from the calculated theoretical values.

The calculated fitting parameter (carrier lifetime) changes versus temperature are shown in Fig. 9. The carrier lifetime increases from 108 ns to 135 ns while temperature decreases from $300 \mathrm{~K}$ to $200 \mathrm{~K}$. According to our calculations the ambipolar carrier diffusion length $L=7.12 \times 10^{-6} \mathrm{~m}$ at $300 \mathrm{~K}$ is comparable to the absorber thickness $7 d=1.07 \times 10^{-6} \mathrm{~m}(7 d / L<1)$.

Percentage share of leakage current increases with decreasing temperature, e.g. at $300 \mathrm{~K}$ the leakage current is $32 \%$ of the total theoretical current flowing through the device, while at $200 \mathrm{~K}$ increases to $72 \%$ (see Fig. 10). At temperature $235 \mathrm{~K}$ leakage current is equal to bulk current. Thus, measured low sensitivity (see Fig. 3) is associated with large leakage currents flowing through the structure.

Figure 11 shows a graph of leakage current $I_{s}$ versus $1 / k_{\mathrm{B}} T$. Activation energy is equal to $80 \mathrm{meV}$. This value is comparable to that obtained in Fig. 6 for low temperatures. These results confirm that: leakage currents have a large impact on the dark current flowing through the structure, especially at low temperatures.
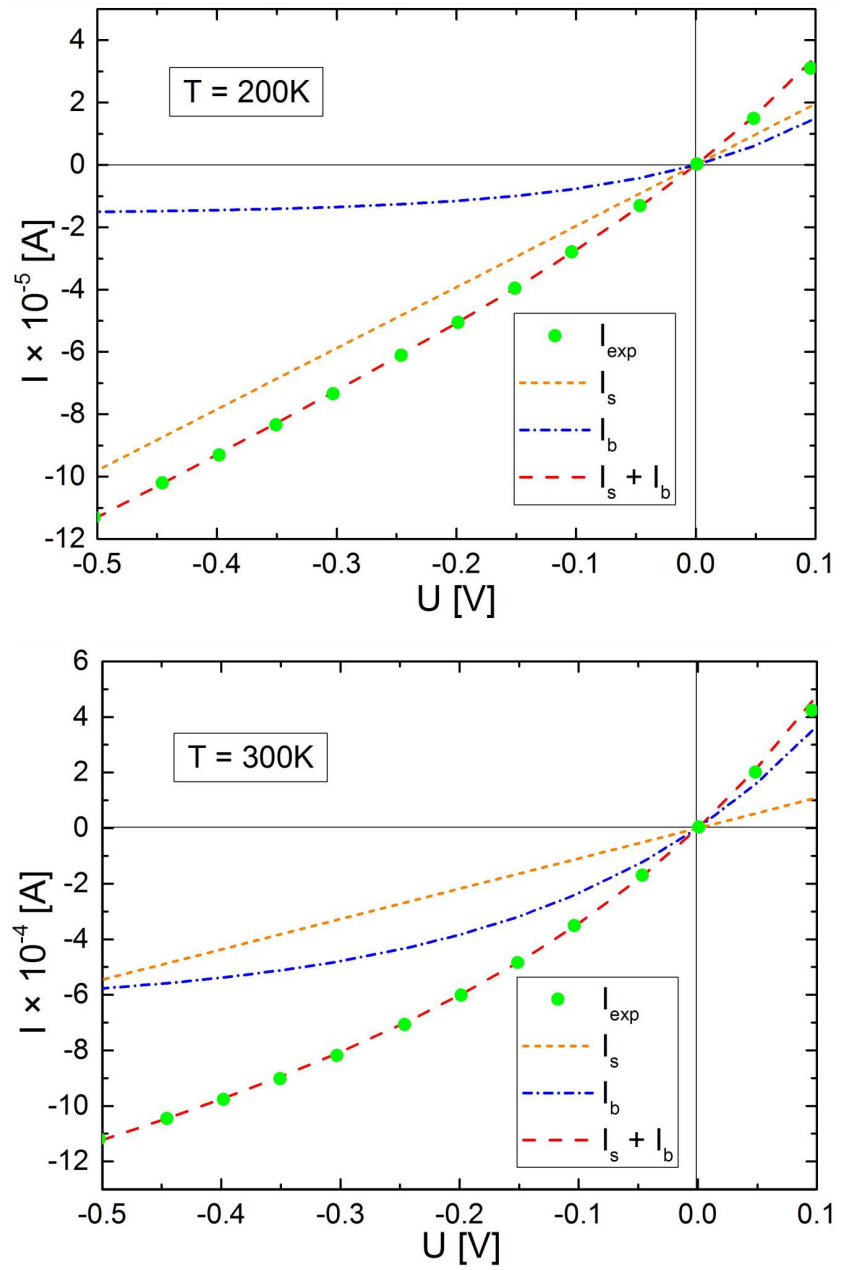

Fig. 8. Measured and modeled dark current versus bias voltage of T2SL InAs/GaSb cascade detector at $200 \mathrm{~K}$ (top) and $300 \mathrm{~K}$ (bottom).

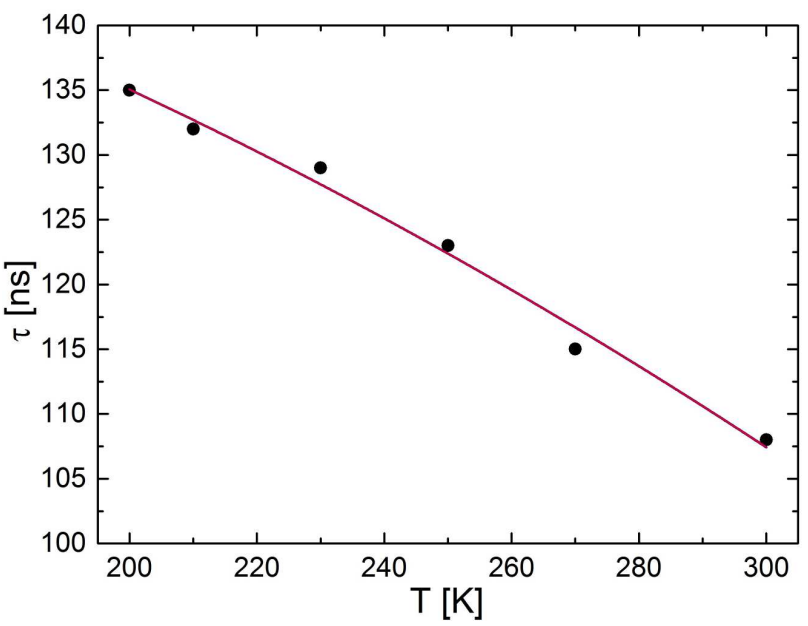

Fig. 9. The carrier lifetime versus temperature. 


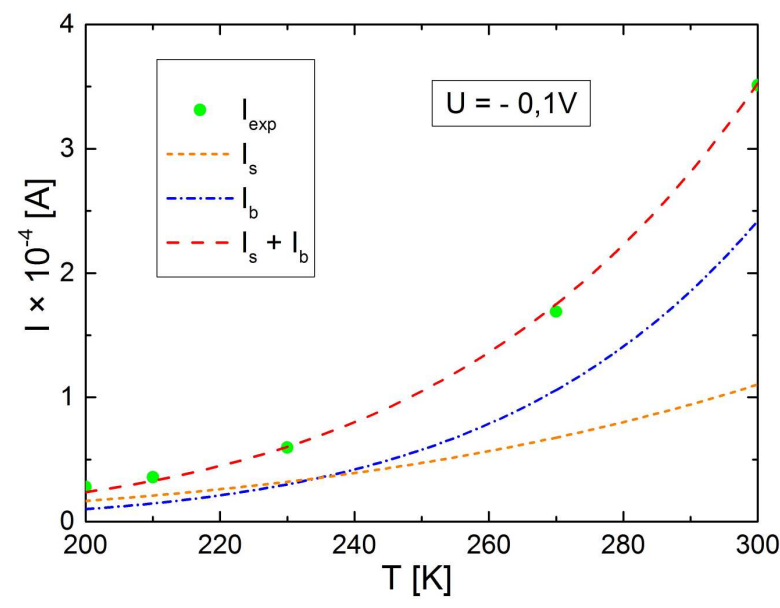

Fig. 10. Measured and modeled dark current versus temperature at applied voltage $-0.1 \mathrm{~V}$.

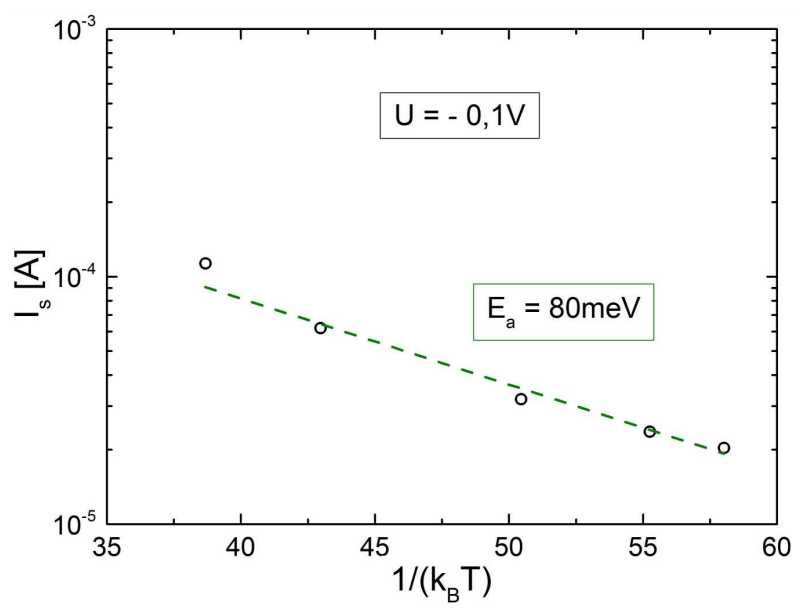

Fig. 11. Characteristic of leakage current versus $1 / k_{\mathrm{B}} T$ at applied voltage $-0.1 \mathrm{~V}$.

\section{Conclusions}

We investigated current-voltage characteristics of interband cascade type-II MWIR InAs/GaSb superlattice photodetector in temperature range from $200 \mathrm{~K}$ to $300 \mathrm{~K}$. Based on the theoretical calculation of dark current treated as a sum of two components: average bulk current and average leakage current we fitted our experimental data to theoretical model, assuming that transport in absorber was determined by dynamics of the intrinsic carriers. Proper fit of the theoretical and experimentally measured characteristics was reached. Based on the fit we estimated carrier lifetime changing from $108 \mathrm{~ns}$ to $135 \mathrm{~ns}$ in the temperature range $300-200 \mathrm{~K}$. The ambipolar carrier diffusion length resulting from the estimated carrier lifetime was comparable to the total absorber thickness. Our result showed that leakage currents have a large impact on the dark current flowing through the structure, especially at low temperatures. Thus, additional channels of leakage current lead to higher dark current levels. Our current work will be concentrated on identifying these additional channels of leakage current.

\section{Acknowledgments}

We acknowledge support by the National Science Centre - the grant no. OPUS/UMO2015/19/B/ST7/02200.

\section{References}

[1] A. Rogalski, Rep. Prog. Phys. 68, 2267 (2005).

[2] S. Mokkapati, C. Jagadish, Mater. Today 12, 22 (2009).

[3] B.J. Sealy, J. IERE. 57, S2 (1987).

[4] R.Q. Yang, Z. Tian, Z. Cai, J.F. Klem, M.B. Johnson, H.C. Liu, J. Appl. Phys. 107, 054514 (2010).

[5] N. Gautam, S. Myers, A.V. Barve, B. Klein, E.P. Smith, D.R. Rhiger, L.R. Dawson, S. Krishna, Appl. Phys. Lett. 101, 021106 (2012).

[6] P. Christol, C. Cervera, R. Chaghi, H. Aït-Kaci, J.B. Rodriguez, L. Konczewicz, S. Contreras, K. Jaworowicz, I. Ribet-Mohamed, Proc. SPIE $\mathbf{7 6 0 8 ,}$ 76081U (2010).

[7] W. Pusz, A. Kowalewski, P. Martyniuk, W. Gawron, E. Plis, S. Krishna, A. Rogalski, Opt. Eng. 53, 043107 (2014).

[8] Y.P. Varshni, Physica 34, 149154 (1967).

[9] R.T. Hinkey, R.Q. Yang, J. Appl. Phys. 114, 104506 (2013).

[10] C. Cervera, J.B. Rodriguez, J.P. Perez, H. Aït-Kaci, R. Chaghi, L. Konczewicz, S. Contreras, P. Christol, J. Appl. Phys. 106, 033709 (2009).

[11] S. Safa, A. Asgari, J. Low Temp. Phys. 181, 223 (2015).

[12] J. Nguyen, D.Z. Ting, C.J. Hill, A. Soibel, S.A. Keo, S.D. Gunapala, Infr. Phys. Technol. 52, 317321 (2009).

[13] P. Martyniuk, A. Rogalski, Infrared Phys. Technol. 70, 125128 (2015). 